\title{
Zeroes of partial sums of the zeta-function
}

\author{
David J. Platt and Timothy S. Trudgian
}

\begin{abstract}
This article considers the positive integers $N$ for which $\zeta_{N}(s)=\sum_{n=1}^{N} n^{-s}$ has zeroes in the half-plane $\Re(s)>1$. Building on earlier results, we show that there are no zeroes for $1 \leqslant N \leqslant 18$ and for $N=20,21,28$. For all other $N$ there are infinitely many such zeroes.
\end{abstract}

\section{Introduction}

The Riemann zeta-function is defined as $\zeta(s)=\sum_{n=1}^{\infty} n^{-s}$ for $\Re(s)>1$. Throughout this article we write the complex variable $s$ as $s=\sigma+i t$ with $\sigma$ and $t$ real, and consider $N$ to be a natural number. Truncation of the zeta-function gives the partial sum $\zeta_{N}(s)=1+2^{-s}$ $+\ldots+N^{-s}$. One may study these partial sums in the hope of deducing some information about $\zeta(s)$. For a comprehensive treatment of these ideas, we refer the reader to [5] and [6].

Turán [16] showed that the Riemann hypothesis would follow if for all $N$ sufficiently large $\zeta_{N}(s)$ had no zeroes in $\sigma>1$. Let $\psi_{N}$ be the supremum over all values of $\sigma$ for which $\zeta_{N}(s)=0$. Montgomery [9] showed that for all $N$ sufficiently large,

$$
\psi_{N}=1+\left(\frac{4}{\pi}-1-o(1)\right) \frac{\log \log N}{\log N},
$$

where the constant $4 / \pi-1$ is best possible. Therefore, for $N$ sufficiently large, $\zeta_{N}(s)$ has zeroes in $\sigma>1$.

Monach [8] made this explicit: for all $N>30$ there are zeroes in $\sigma>1$. His proof was in two parts: an analytic argument for $N \geqslant 549,798$ and a computational proof for $30<N<549,798$. The latter proof is contained in [8, Lemma 3.14, pp. 134-135]. Monach's work can be combined with the results of Turán and Spira to give Table 1 below.

Indeed, van de Lune and te Riele [17] actually computed some zeroes of $\zeta_{N}(s)$ for $N=$ 19, 22-27, 29-35, 37-41, 47. Adapting Bohr's theorems on values assumed by Dirichlet series, Spira [14, Theorem 3] (see also [15, p. 163]) showed that if $\zeta_{N}(s)$ has one zero in $\sigma>1$, then it has infinitely many such zeroes.

Therefore, all that remains is to investigate whether, for

$$
N \in\{10,11,12,13,14,15,16,17,18,20,21,28\}
$$

$\zeta_{N}(s)$ has zeroes in $\sigma>1$. We find that there are no zeroes for each of these values of $N$. Combining this with Table 1, one proves the following theorem.

TheOrem 1.1. For $1 \leqslant N \leqslant 18$ and $N=20,21,28$ there are no zeroes of $\zeta_{N}(s)$ in the region $\sigma>1$; for all other positive $N$ there are infinitely many such zeroes.

Received 6 July 2015; revised 23 November 2015.

2010 Mathematics Subject Classification 11M06 (primary), 11Y35 (secondary).

The second author was supported by the Australian Research Council DECRA Grant DE120100173. 
TABLE 1. Zeroes of $\zeta_{N}(s)$ in $\sigma>1$ for various values of $N$.

\begin{tabular}{cc}
\hline Range of $N$ & Are there zeroes of $\zeta_{N}(s)$ in $\Re(s)>1 ?$ \\
\hline $1-5$ & No, $[\mathbf{1 6}$, pp. $7-8]$ \\
$6-9$ & No, $[\mathbf{1 3}$, p. 550$]$ and $[\mathbf{1 5}$, Table II, $\S 4]$ \\
19 & Yes, $[\mathbf{1 5}$, Table III, $\S 4]$ \\
$22-27$ & Yes, $[\mathbf{1 5}$, Table III, $\S 4]$ \\
$29-50$ & Yes, $[\mathbf{1 5}$, Table III, $\S 4]$ \\
$\geqslant 51$ & Yes, $[\mathbf{8}$, Theorem 3.8] \\
\hline
\end{tabular}

\section{Numerical computation}

\subsection{Interval arithmetic}

Almost all real numbers are not exactly representable by any finite-precision, floating-point system such as the 64-bit IEEE implementation available on most modern processors. Thus, any computation involving such a floating-point system will, unless we are very lucky, only produce an approximation to the true result. One way of managing this is to use interval arithmetic (see, for example, [10] for a good introduction). Instead of storing a floating-point number that is an approximation to the value we want, we store an interval bracketed by two floating-point numbers that contains the true value.

Interval arithmetic has been used to manage the accumulation of round-off and truncation errors. In this paper, we exploit the technique to get zero-free regions rigorously. As an example, consider the function $f: \mathbb{R} \rightarrow \mathbb{R}$ defined by

$$
f(x)=x^{2}-4 x+3
$$

Suppose we wish to demonstrate that $f$ has no zeroes for $x \in[4,5]$. Then we can compute

$$
f([4,5])=[16,25]-[16,20]+3=[-1,12] .
$$

Since this is inconclusive, we try again, but this time with the interval split in two. We have

$$
f([4,4.5])=[16,20.25]-[16,18]+3=[1,7.25]
$$

and

$$
f([4.5,5])=[20.25,25]-[18,20]+3=[3.25,10]
$$

and we have our result ${ }^{\dagger}$.

\subsection{Description of algorithm}

We first note that we need not search in all of $\sigma>1$ to find zeroes of $\zeta_{N}(s)$. Spira [13, Theorem 1] proved that all zeroes of $\zeta_{N}(s)$ must have real part less than 1.85; this was sharpened in [3, Theorem 3.1] to 1.73 . We therefore need only consider $\sigma \in(1,1.73]$. We can improve this for some values of $N$, but, as we shall see in $\S 2.3$, this is more than sufficient for our purposes.

\footnotetext{
${ }^{\dagger}$ Note that if we had written $f(x)=(x-1)(x-3)$, then $f[4,5]=[3,4] \cdot[1,2]=[3,8]$, which is the 'correct' result. This sensitivity is common in expressions involving intervals.
} 
Let us consider the case $N=28$. Let $p$ denote a prime and let $\theta_{p}=t \log p$. Hence, we have

$$
\zeta(\sigma+i t)=1+\frac{\exp \left(-i \theta_{2}\right)}{2^{\sigma}}+\frac{\exp \left(-i \theta_{3}\right)}{3^{\sigma}}+\frac{\exp \left(-i 2 \theta_{2}\right)}{4^{\sigma}}+\ldots+\frac{\exp \left(-i\left(2 \theta_{2}+\theta_{7}\right)\right)}{28^{\sigma}}
$$

and we will now write $\zeta_{28}\left(\sigma, \theta_{2}, \ldots, \theta_{23}\right)$ for $\zeta_{28}(\sigma+i t)$ under such a change of variables.

It would appear that we need to examine the space $\sigma \in(1,1.73], \theta_{p} \in[0,2 \pi)$ for $p \leqslant 23$, for zeroes. In fact, we can do considerably better. First, we observe that $\theta_{17}, \theta_{19}$ and $\theta_{23}$ only appear once in the sum. Call the sum without those three terms $\zeta_{28^{\prime}}\left(\sigma, \theta_{2}, \ldots, \theta_{13}\right)$. Then $\zeta_{28}$ cannot have a zero if there is no $\sigma, \theta_{2}, \ldots, \theta_{13}$ such that

$$
\left|\zeta_{28^{\prime}}\left(\sigma, \theta_{2}, \ldots, \theta_{13}\right)\right| \leqslant 17^{-\sigma}+19^{-\sigma}+23^{-\sigma} .
$$

We can go further. The $\theta_{11}$ and $\theta_{13}$ terms only appear on their own or in conjunction with $\theta_{2}$. We write $a=11^{-\sigma}, b=22^{-\sigma}, c=13^{-\sigma}$ and $d=26^{-\sigma}$. Then a little high-school geometry (the cosine rule to be precise) tells us that

$$
\left|\frac{\exp \left(-i \theta_{11}\right)}{11^{\sigma}}+\frac{\exp \left(-i\left(\theta_{11}+\theta_{2}\right)\right)}{22^{-\sigma}}\right| \leqslant \sqrt{a^{2}+b^{2}+2 a b \cos \theta_{2}}
$$

and

$$
\left|\frac{\exp \left(-i \theta_{13}\right)}{13^{\sigma}}+\frac{\exp \left(-i\left(\theta_{13}+\theta_{2}\right)\right)}{26^{-\sigma}}\right| \leqslant \sqrt{c^{2}+d^{2}+2 c d \cos \theta_{2}} .
$$

Call $\zeta_{28^{\prime \prime}}\left(\sigma, \theta_{2}, \theta_{3}, \theta_{5}, \theta_{7}\right)$ the result obtained by removing the $n=11,13,22$ and 26 terms from $\zeta_{28^{\prime}}$. With $a, b, c$ and $d$ as above, define

$$
f\left(\sigma, \theta_{2}\right)=17^{-\sigma}+19^{-\sigma}+23^{-\sigma}+\sqrt{a^{2}+b^{2}+2 a b \cos \theta_{2}}+\sqrt{c^{2}+d^{2}+2 c d \cos \theta_{2}} .
$$

Then $\zeta_{28}$ cannot have a zero if there is no $\sigma, \theta_{2}, \ldots, \theta_{7}$ such that

$$
\left|\zeta_{28^{\prime \prime}}\left(\sigma, \theta_{2}, \theta_{3}, \theta_{5}, \theta_{7}\right)\right| \leqslant f\left(\sigma, \theta_{2}\right) .
$$

Our algorithm is as follows. Divide $\sigma, \theta_{2}, \theta_{3}, \theta_{5}$ and $\theta_{7}$ into small intervals that cover $[1,1.73]$ and $[0,2 \pi]^{4}$, respectively. We refer to any choice of five such intervals as a 'box'. Push all possible boxes onto the stack. While the stack is not empty, pop off a box and compute an interval $z$ containing $\left|\zeta_{28^{\prime \prime}}\right|$ for that box. Compute an interval containing $f\left(\sigma, \theta_{2}\right)$. If the interval $z-f\left(\sigma, \theta_{2}\right)$ is wholly positive, then that box did not contain any zeroes, so discard it. If the interval is wholly negative, then terminate with failure ${ }^{\dagger}$. If the interval straddles zero, then divide the box into 16 smaller boxes by halving the intervals for the $\theta_{p}$, and push these new boxes onto the stack.

\subsection{Details of the implementation}

We implemented this algorithm in ' $\mathrm{C}++$ ' using our own double-precision interval package written in assembler. This exploits an idea of Lambov [7] to make efficient use of the SSE instruction set of modern processors and uses CRMLIB [11] to implement the transcendental functions.

We divided the interval for $\sigma$ into 16 sub-intervals $\left[1+\left(2^{n}-1\right) \cdot 2^{-16}, 1+\left(2^{n+1}-1\right) \cdot 2^{-16}\right]$ for $0 \leqslant n \leqslant 15$. Therefore, the first interval checked was $\sigma=\left[1,1+2^{-16}\right]$ and the last ${ }^{\ddagger}$ was

\footnotetext{
${ }^{\dagger}$ We believe that this condition indicates the presence of infinitely many zeroes. We are grateful to a referee for suggesting a means by which one might seek to establish this, based on $[\mathbf{2}, \mathbf{4}, \mathbf{1 2}]$. However, the weaker statement is sufficient for our purposes and we do not pursue this line of thought further.

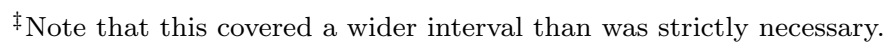


$\sigma=\left[3 / 2-2^{-16}, 2-2^{-16}\right]$. Each of these intervals for $\sigma$ was handled by a single core of a compute node of the University of Bristol's Bluecrystal Phase III cluster [1 $]^{\S}$. Within a single core, the intervals for $\theta_{2}, \theta_{3}, \theta_{5}$ and $\theta_{7}$ were initially divided into $16,8,4$ and 2 sub-intervals, respectively, for a total of 1024 boxes. Since $\theta_{2}$ contributed to more terms than the other variables, it made sense to start with a narrower search here: this seemed to work well in practice.

Table 2 shows the data for $N=28$ and $\sigma \in\left[1,1+2^{-16}\right]$. At each iteration, a box could result in 16 new boxes; at first this is what we see. We see that after the second iteration, the remaining search space decreases dramatically.

TABLE 2. Number of boxes at each iteration for $N=28$ and $\sigma \in\left[1,1+2^{-16}\right]$.

\begin{tabular}{ccc}
\hline Iteration & Number of boxes & Coverage $(\%)$ \\
\hline 1 & 1024 & 100 \\
2 & 16256 & 99.2 \\
3 & 45920 & 17.5 \\
4 & 118560 & 2.83 \\
5 & 170048 & 0.25 \\
6 & 195920 & 0.018 \\
7 & 212960 & 0.0012 \\
8 & 82016 & 0.000030 \\
\hline
\end{tabular}

We ran this algorithm for those $N$ in (1.1) and in every case confirmed that $\zeta_{N}(s)$ has no zeroes for $\sigma \geqslant 1$. Checking each $N$ took much less than a minute of elapsed time using 16 cores, with $N=21$ taking the longest at $30 \mathrm{~s}$.

Acknowledgements. We are grateful to Andrew Booker with whom we discussed several aspects of this problem and to the referees for their careful reading of the manuscript.

\section{References}

1. ACRC, BlueCrystal User Guide, 2015, https://www.acrc.bris.ac.uk/pdf/bc-user-guide.pdf.

2. C. E. Avellar and J. K. Hale, 'On the zeros of exponential polynomials', J. Math. Anal. Appl. 73 (1980) 434-452.

3. P. Borwein, G. Fee, R. Ferguson and A. van der WaAll, 'Zeros of partial sums of the Riemann zeta function', Exp. Math. 16 (2007) no. 1, 21-39.

4. E. Dubon, G. Mora, J. M. Sepulcre, J. I. Ubeda and T. Vidal, 'A note on the real projection of partial sums of Riemann zeta function', RACSAM 108 (2014) 317-333.

5. S. M. Gonek and A. H. LedoAn, 'Zeros of partial sums of the Riemann zeta-function', Int. Math. Res. Not. IMRN 2010 (2010) no. 10, 1775-1791.

6. S. M. GoneK and H. L. Montgomery, 'Zeros of a family of approximations of the Riemann zeta-function', Int. Math. Res. Not. IMRN 2013 (2013) no. 20, 4712-4733.

7. B. LAmbOv, 'Interval arithmetic using SSE-2', Reliable implementation of real number algorithms: theory and practice, Lecture Notes in Computer Science (ed. P. Hertling et al.; Springer, Berlin, 2008).

8. W. R. MONACH, 'Numerical investigation of several problems in number theory', PhD Thesis, University of Michigan, 1980.

9. H. L. Montgomery, 'Zeros of approximations to the zeta function', Studies in pure mathematics: to the memory of Paul Turán (ed. P. Erdős; Birkhäuser, Basel, 1983) 497-506.

10. R. E. Moore, Interval analysis (Prentice-Hall, Englewood Cliffs, NJ, 1966).

11. J. M. MulLeR, 'Correctly rounded mathematical library', http://lipforge.ens-lyon.fr/www/crlibm/.

12. J. M. Sepulcre and T. Vidal, 'A new approach to obtain points of the closure of the real parts of the zeros of the partial sums $1+2^{z}+\ldots+n^{z}, n \geqslant 2$ ', Kybernetes 41 (2012) no. 1, 96-107.

13. R. SpIRA, 'Zeros of sections of the zeta function I', Math. Comp. 20 (1966) 542-550.

$\S$ A single node of Phase III contains two eight-core Intel E5-2670 Sandy Bridge processors running at $2.6 \mathrm{GHz}$. 
14. R. SPIRA, 'Sets of values of general Dirichlet series', Duke Math. J. 35 (1968) 79-82.

15. R. SpIRA, 'Zeros of sections of the zeta function II', Math. Comp. 22 (1968) 168-173.

16. P. Turán, 'On some approximative Dirichlet-polynomials in the theory of the zeta-function of Riemann', Danske Vid. Selsk. Math.-Fys. Medd. 24 (1948) no. 17, 1-36.

17. J. VAN DE LUNe and H. J. J. TE RIELE, 'Numerical computation of special zeros of partial sums of Riemann's zeta function', Computational methods in number theory, part II, Mathematical Centre Tracts 155 (Mathematisch Centrum, Amsterdam, 2010) 371-387.

David J. Platt

Heilbronn Institute for

Mathematical Research

University of Bristol

Bristol

United Kingdom

dave.platt@bris.ac.uk
Timothy S. Trudgian

Mathematical Sciences Institute

The Australian National University

ACT 0200

Australia

timothy.trudgian@anu.edu.au 